\title{
Valeurs familiales de sujets féminins novateurs : perspectives d'avenir
}

\section{The Family Values of Innovating Feminine Subjects : Perspectives of the Future Palores familiares de sujetos femeninos innovadores : perspectivas de futuro}

\section{Colete CARISSE et Joffre DUMAZEDIER}

Volume 2, numéro 2, novembre 1970

URI : https://id.erudit.org/iderudit/001122ar

DOI : https://doi.org/10.7202/001122ar

Aller au sommaire du numéro

Éditeur(s)

Les Presses de l'Université de Montréal

ISSN

0038-030X (imprimé)

1492-1375 (numérique)

Découvrir la revue

Citer cet article

CARISSE, C. \& DUMAZEDIER, J. (1970). Valeurs familiales de sujets féminins novateurs : perspectives d'avenir. Sociologie et sociétés, 2(2), 265-282. https://doi.org/10.7202/001122ar

\section{Résumé de l'article}

Le présent article essaie d'esquisser les tendances du changement familial dans une société technologiquement avancée. Les données ont été recueillies auprès de cent cinquante femmes jugées novatrices dans divers secteurs d'activité. Nous leur avons demandé : 1) Que pensez-vous de la famille comme institution ? 2) Quelle serait la relation idéale parents-enfants ? 3) Que pensez-vous du mariage ? 4) Quelle serait la relation idéale entre un homme et une femme? Les résultats ont été regroupés à partir de trois points de référence qui correspondent aux trois types de société : traditionnelle, industrielle et postindustrielle. Près des deux tiers des mentions définissent une vie familiale qui relève de la société traditionnelle ou industrielle. Par contre, les autres ou bien rejettent ces définitions ou bien se situent en deçà indiquant des dominantes de la vie familiale ou conjugale en devenir. 


\title{
Valeurs familiales de sujets féminins novateurs
}

\author{
Perspectives d'avenir *
}

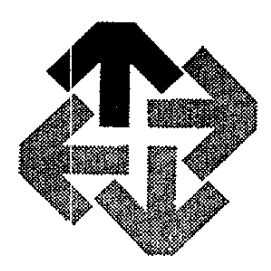

COLETTE CARISSE et JOFFRE DUMAZEDIER

\section{APPROCHE GÉNÉRALE}

Nous PRÉSENTONS dans cet article les valeurs familiales de femmes jugées innovatrices dans différents secteurs d'activité. Nous espérons trouver dans leur discussion de la famille et du mariage des éléments de la famille future ${ }^{1}$. Mais pour identifier ces dimensions nouvelles, nous avons besoin d'un profil précis des différents types de société dont la famille est une partie constituante. Nous sommes d'avis que la dichotomie : traditionnel $v s$ moderne, ne peut rendre compte de la réalité familiale dans une société qui a déjà atteint un niveau élevé de développement technologique et où de nouveaux modèles culturels sont sécrétés à un rythıme accéléré.

La modernité est, le plus souvent, opposée à une mentalité ou à des structures traclitionnelles ${ }^{2}$. Dans les recherches d'Alex Inkeles, par exemple, la mentalité

* Les données présentées ici font partie d'une recherche plus vaste sur l'innovation sociale et culturelle de sujets féminins et québécois : l'élaboration théorique que nous présentons est donc forcément incomplète. Cette recherche est financée par un octroi du Conseil des Arts du Canada.

1. Nous supposons dans la présente analyse que l'innovativité est une disposition de la personne en situation favorable. Toutefois, cette affirmation globale reste une hypothèse à être confirmée dans la recherche générale.

2. Voir entre autres exemples : Myron Weiner (édit.), Modernization, New York, Basic Books, 1966. 
préindustrielle est opposée à certaines caractéristiques de l'homme moderne, notamment l'ouverture aux idées nouvelles, à l'information, l'habileté à prendre des décisions et à exprimer des opinions, la conscience de pouvoir planifier et un souci de l'efficacité. Le paysan devra acquérir ces traits pour fonctionner adéquatement dans sa société « en voie de développement » vers l'industrialisation. Toutefois, pour qualifier les sociétés (ou plutôt des régions de ces sociétés) qui ont déjà atteint un taux de développement technologique et industriel élevé, le terme moderne est trop vague : ce terme se réfère à la fois à un type de société basé sur l'urbanisation et l'industrialisation et aux éléments qui émergent de cette société une fois qu'un certain palier a été atteint. Pour arriver à plus de rigueur, il faut distinguer différents aspects : technologique et économique, social et culturel, de même qu'éthique. L'hypothèse sous-jacente à l'élaboration d'un modèle de société postindustrielle est que, passé un seuil minimum de développement technologique et économique, s'accomplissent des mutations culturelles et sociales qui donnent naissance à un nouveau type de société qu'on peut qualifier simplement de postindustrielle ou de postmoderne.

Certains auteurs parlent de société néo-technique, pour montrer la différence essentielle entre la machine moderne et l'outil ou l'engin qui, depuis des millénaires, prolongent la dextérité ou la force physique de l'homme. Les nouveaux développements technologiques sont basés sur la chimisation qui synthétise les structures naturelles et sur l'automation qui, par le processus cybernétique, prolonge le cerveau humain et possède la propriété de rectifier, voire même de décider, les étapes d'un parcours. Cette technologie se reflète dans la distribution des occupations; du primaire on était passé au secondaire pour aboutir à la priorité du tertiaire. On parle de plus en plus de l'importance du quaternaire, c'est-à-dire de la production et de la distribution de l'information prise au sens large de connaissance et de communication.

Du point de vue économique, le développement reste lié à l'accroissement industriel. Les problèmes techniques de la subsistance sont théoriquement résolus et la pauvreté est avant tout un problème politique de distribution équitable des ressources. La production des biens n'est plus exclusivement orientée vers la reproduction des forces productrices du travail : elle devient fin en soi et, pour favoriser la croissance illimitée, des besoins nouveaux sont créés. C'est la base de la société dite de consommation : toutefois la quantité des biens produits grâce à la nouvelle technologie affecte la qualité même de la vie, le style dans l'art de se vêtir, de se nourrir et de se loger. Plus de biens et de services sont produits en moins de temps. A long terme, on peut donc parler de temps libéré pour être si possible utilisé à des fins librement orientées. C'est la civilisation du loisir, au sens le plus large du terme ${ }^{3}$.

Le développement technique et économique est tributaire des nouveaux modes de penser : la révolution scientifique est aussi révolution culturelle, et pour plusieurs auteurs science et culture deviennent synonymes ${ }^{4}$. Le style cognitif de l'homme qui dialogue avec un cerveau électronique est différent de l'attitude

3. Optique développée par J. Dumazedier dans Vers une civilisation du loisir, Paris, Le Seuil, 1962.

4. Jacques Bureau a défini brillamment ce nouveau style de penser et ses conséquences sociales et culturelles dans l'Ere logique, Paris, Laffont, 1969. 
de l'homme qui commande une machine. Le raisonnement analogique basé sur l'évaluation probabiliste déplace la logique cartésienne basée sur la déduction. C'est la culture en mosaïque chère à McLuhan, qui remplace la culture linéaire. Les auteurs les plus différents s'entendent pour reconnaître l'innovation dans la création de relations nouvelles entre des éléments connus d'information ${ }^{5}$. L'innovation et le changement sont valorisés; c'est cette valorisation même qui pousse le leader à une adoption hâtive de l'innovation et à sa diffusion ${ }^{\theta}$.

Socialement, c'est l'accès à la science et à l'information qui devient source privilégiée de pouvoir. Les conflits de classe sont définis moins en termes de position en regard des moyens de production que de distance de la source de connaissance et d'information. Les barrières culturelles à la mobilité sont plus difficiles à franchir que les barrières économiques ?. C'est la société de masse qui isole l'expert. Les décisions programmées exercent une emprise de plus en plus rigoureuse sur l'homme moyen. Cette société contrôle non seulement la nature et la matière, mais par son organisation, elle contrôle l'homme.

Sur le plan éthique, la valorisation de la créativité mène au rejet de l'autoritarisme sous toutes ses formes : dans la transmission du savoir comme dans la définition politique des buts collectifs. Les critères de choix remplacent les principes inculqués, tout comme sur le plan scientifique la probabilisation remplace la déduction. Face à la force contraignante du pouvoir, les individus et les groupes revendiquent le droit à la participation d'une part, et, d'autre part, le droit à la jouissance, au rêve, à l'éros, à la fête, à l'expression de soi ${ }^{8}$. Allégé en partie des contraintes de subsistance, l'individu revendique le droit à des conduites autonomes non réglées socialement et moralement, dans des domaines aussi divers que la religion, l'amour, l'art, le sport. C'est la redécouverte du corps, de l'environnement, de la nature, de l'univers spirituel.

Les sources de conflits sont donc puissantes entre la société technocratique qui programme l'avenir des sociétés et des groupes d'une part, et le désir de

5. Le processus créateur se définit pour moi comme étant l'émergence dans l'action d'un produit relationnel nouveau, qui se détache de la nature unique de l'individu d'une part, et des événements, des personnes ou des circonstances de sa vie d'autre part * (Carl R. Rogers, le Développement de la personne, trad., Paris, Dunod, 1968, p. 247). (Innovation consists of organizing reality into relationships embodying new mental or aesthetic concepts, the new relationships serving the purpose of the innovator better than the old; [...] more striclly defined, innovation is performing activity that involves problem solving in a high degree. $>$ (Everett E. Hagen, On the Theory of Social Change, Homewood (IIl.), The Dorsey Press, 1962, p. 86). Cet auteur fait de l'innovation le moteur du développement économique. - La créativité est cette aptitude particulière de l'esprit de réarranger les éléments du champ de conscience d'une façon originale et susceptible de donner lieu à des opérations dans un quelconque champ phénoménal. (Abraham Moles, Sociodynamique de la culture, Paris, Mouton, 1967, p. 91).

6. C'est la conclusion d'une recherche de Marshall $\mathrm{H}$. Becker : \& It would appear that when the norms of a particular social group favor change, progressive behavior will be located in group leaders; but if the norms favor maintenance of the status quo, the leaders will retain a conservative approach, while marginals will assume the role of innovators. * (* Sociometric Location of Innovativeness : Reformulation and Extension of the Diffusion Model s, American Sociological Review, vol. 35, avril 1970, p. 268).

7. Thèse soutenue par A. Touraine dans la Société post-industrielle, Paris, Editions Dencël, * Bibliothèque médiations $», 1969$.

8. Cette révolution éthique, parallèle à la révolution scientifique, a été analysée de façon émouvante (quand on connaît la suite des événements) par une équipe pluridisciplinaire de l'académie des sciences de Tchécoslovaquie. Voir Radovan Richta, la Civilisation au carrefour, trad., Paris, Editions Anthropos, 1968. 
participation et le rejet de l'autorité linéaire d'autre part. Pour l'individu, l'équilibre peut être très pénible entre la nécessité de participer à une société active et le désir de s'adonner à des activités libres qui ont pour fin l'individu lui-même. Ces mêmes conflits, on peut aussi s'attendre à les retrouver au cœur de la vie familiale, lieu privilégié où l'on vit sa société.

Dans cette introduction nous avons présenté un résumé des analyses de macrosociologues. Il s'agit, bien sûr, d'une construction de l'esprit sur la base de données empiriques éparses. Mais comme tout type idéal, c'est un modèle qui permet d'ordonner les éléments de la réalité sociale et de leur donner un sens. Aux deux types connus de traditionnel et d'industriel, nous ajouterons un troisième point de référence, à savoir postindustriel ${ }^{9}$. Si l'hypothèse d'une société postindustrielle est juste, nous devrions retrouver dans les définitions de famille et de mariage idéals, des éléments qui ne s'accordent pas avec l'éthique protestante qui valorise le travail, le succès, l'autorité et l'individualité. Ces dimensions nouvelles permettraient de parler non plus de désintégration de la famille mais bien d'une redéfinition, qui est partie intégrante de l'élaboration d'un nouveau type de société ${ }^{10}$. Mais avant de décrire nos résultats, nous justifierons leur valeur indicative en termes de prospective.

\section{MÉTHODOLOGIE}

Nous avons proposé l'éclatement de la notion de moderne en deux types successifs. Nous sommes donc justifiés de rechercher nos données dans une société technologiquement avancée ${ }^{11}$. La province de Québec, plus particulièrement la région de Montréal, répond à ce critère quels que soient les indicateurs utilisés : le revenu atteint 2000 par tête, près d'un tiers de la population atteint ou dépasse treize années d'études, quatre personnes sur cinq vivent en milieu urbain dont la moitié dans des villes de plus de 40000 habitants, plus de la moitié de la population active se retrouve dans le secteur tertiaire ou quaternaire, la quasi-totalité des foyers possède au moins une télévision et la semaine de travail pour les employés de bureau est de trente-sept heures par semaine ${ }^{12}$.

Les sujets interrogés sont des femmes jugées innovatrices dans différents secteurs d'activité; il s'agit tout aussi bien d'une directrice de prison que d'une mathématicienne ou d'une escrimeuse, d'un membre actif d'un mouvement

9. Afin de respecter les limites imposées à un article, nous n'avons pas décrit systématiquement les types idéaux de société traditionnelle et industrielle. Ces descriptions sont d'ailleurs connues.

10. Harold Feldman oppose les thèses de désintégration et d'adaptation de la famille. Il réfute la thèse de la désintégration et opte pour une interprétation d'adaptation. L'agent clef des changements dans les structures familiales est, selon lui, la redéfinition du statut de la femme (cf. Conférence à l'Institut Vanier de la famille, Ottawa, avril 1970).

11. Ce qui ne veut pas dire que des indications d'un nouveau type de société ne peuvent être recherchées en société traditionnelle; la preuve d'un passage obligatoire par un type de société industriel est loin d'être faite. D'ailleurs, le profil de développement d'une société, selon les secteurs, n'est pas nécessairement consistant. Nous ne voulons pas ici développer ce problème.

12. Données colligées à partir des sources officielles par $\mathrm{M}$. Laplante, le Développement culturel au Québec, thèse de $3^{e}$ cycle, Paris, 1969. 
contestataire que d'une présidente d'association familiale ${ }^{13}$. Les 150 personnes interrogées ont été sélectionnées, selon les secteurs, par trois juges à partir d'une liste d'environ 1000 noms recueillis de différentes sources : informateur clef, télévision, journaux, association. Nous avions défini comme innovatrice une personne qui, face à sa critique de la situation, peut inventer et réaliser un projet jugé meilleur que la situation antérieure selon des critères de participation active ou de développement de la personne.

Nous aurions pu recueillir nos informations auprès d'un échantillon représentatif de la population générale. Mais à moins d'un échantillon très considérable nous n'aurions pu que difficilement isoler du comportement, ou attitude modale, la déviation positive, marginale, que constitue la création de modèles culturels nouveaux, car la déviation est nécessairement minoritaire. De plus, nous avons plus de chances de trouver cette déviation positive, selon l'expression de Buckley, auprès de sujets innovateurs à cause de leur niveau plus élevé de conscience sociale. Ils sont, par définition, plus lucides devant la réalité sociale et plus conscients des valeurs qui les orientent : les projets déjà réalisés et pour lesquels ils sont reconnus sont une réponse consciente à la réalité ${ }^{14}$. Les novateurs sont porteurs d'avenir possible, dans la mesure où les modèles qu'ils proposent sont diffusés et perçus comme un choix possible entre des alternatives d'action, tant par les individus que par les politiques ${ }^{15}$. Nous reviendrons sur ce point dans la conclusion.

La recherche principale, dont sont tirées les données présentées ici, étudie le système d'action des innovatrices perçu comme une intervention active dans une société donnée. À la fin de l'entrevue, nous avons posé quatre questions ouvertes, non structurées : 1) Que pensez-vous de la famille comme institution? 2) Quelle serait, selon vous, la relation idéale entre parents et enfants? 3) Que pensez-vous du mariage ? 4) Quel serait, selon vous, la relation idéale entre homme et femme ? Le texte des entrevues (moyenne de trois heures par entrevue) ayant été transcrit, nous avons isolé tout énoncé traitant de ces questions dans l'ensemble de l'entrevue. Dans une première lecture, nous avons dressé une liste des item d'information et nous avons établi un lexique des termes utilisés (i.e. mots clefs dans la description d'une situation). Nous avons tenté d'ordonner ces termes sur des axes à trois points de référence qui correspondent aux trois types de société : traditionnelle, industrielle et postindustrielle (voir tableau 1). Les unités d'information ont alors été identifiées, en évitant la redondance dans une même entrevue, puis codées en

13. La répartition selon les secteurs est la suivante : producteurs de modèles culturels : scientifiques (10), artistiques (35), sportifs (5); communication des modèles : enseignemen: (14), communication de masse (17); économie : production (7), distribution (7), syndicalisme (6); activités : sociale (20), familiale (13), religieuse (5), politique (5); expression personnelle (16).

14. A. Etzioni, The Active Society, New York, Free Press, 1968, p. 239-240 : * The reacitions of the more conscious actors are expected to be more a creative * because these actors are more reflective and take more factors into account, i.e. are aware of more conditions' and options [...] we expect them to be more transformable and more utopian [...] They are capable of transcending themselves which requires an ability to design new patterns and to direct efforts towards their realization." L'idée d'une conscience axiologique a été développée par Raymond Polin, la Compréhension des valeurs, Paris, Presses Universitaires de Frarice, * Bibliothèque de philosophie contemporaine ", 1945.

15. W. Buckley, * Society as a Complex Adaptive System », dans Modern Systems Research, Chicago, Aldine Publishing, 1968, p. 495 : * A requisite of sociocultural systems is the development and maintenance of a significant level of non-pathological deviance manifest as a pool of alternate ideas and behaviors with respect to the traditional institutionalized ideologies and role behaviors. 
TABLEAU 1

Termes d'identification

\begin{tabular}{|c|c|c|}
\hline famille traditionnelle & $\begin{array}{l}\text { famille } \\
\text { en société industrielle }\end{array}$ & $\begin{array}{c}\text { famille } \\
\text { en société postindustrielle }\end{array}$ \\
\hline $\begin{array}{l}\text { cellule de base } \\
\text { prédominance de la famille }\end{array}$ & $\begin{array}{l}\text { lieu de ressourcement } \\
\text { privatisation }\end{array}$ & $\begin{array}{l}\text { lieu d'expression } \\
\text { réseau ouvert } \\
\text { de communication }\end{array}$ \\
\hline satisfaction morale & revitalisation & gratification \\
\hline devoir & contrat & affinité \\
\hline statut & individu & personne \\
\hline fortement structuré & équilibre fonctionnel & développement spontané \\
\hline $\begin{array}{l}\text { spécificité des tâches } \\
\text { dans la famille }\end{array}$ & $\begin{array}{l}\text { échange fonctionnel : } \\
\text { instrumental vs affectif }\end{array}$ & $\begin{array}{l}\text { participation collective } \\
\text { intra-familiale et } \\
\text { extra-familiale }\end{array}$ \\
\hline autorité forte & démocratie & leadership selon compétence \\
\hline ignorance des options & planification & spontanéité \\
\hline socialisation dirigée & développement individuel & $\begin{array}{l}\text { différence et échange } \\
\text { intergénérationnels }\end{array}$ \\
\hline adulte-étalon & adulte-ressource & adulte-apprenti \\
\hline $\begin{array}{l}\text { présence nécessaire } \\
\text { des parents }\end{array}$ & disponibilité des parents & vie autonome \\
\hline don de soi & liberté dans l'amour & $\begin{array}{l}\text { actualisation de soi } \\
\text { dans la relation }\end{array}$ \\
\hline monologue & dialogue & échange significatif \\
\hline
\end{tabular}

TABLEAU 2

La famille en tant qu'institution

\begin{tabular}{lll}
\hline dimensions valorisées & fréquence dimensions rejetées & fréquence \\
\hline
\end{tabular}

\section{Fonctions traditionnelles de la vie familiale}

la famille : valeur de base dans la société

la famille : une valeur désuète

la famille : cellule première de la société

la famille : essentielle

à la procréation

4

8

la famille : essentielle

à la socialisation des enfants

$\begin{array}{cc}8 & \begin{array}{l}\text { le droit de ne pas avoir d'enfants } \\ \text { existence d'autres agents } \\ \text { de socialisation }\end{array} \\ \frac{6}{35} & \begin{array}{l}\text { la non-prépondérance du rôle } \\ \text { maternel pour les femmes }\end{array}\end{array}$
total

non-prépondérance du rôle
maternel pour les femmes

2. Fonctions de la famille en société industrielle

la famille : refuge face à un monde de compétition

la famille : source d'affectivité

la famille : compromis entre

les intérêts des membres

nécessité d'une

planification familiale

total

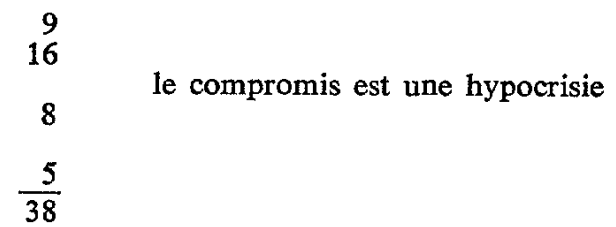

cions nouvelles de la famille lieu de relations significatives nécessité de créer rejet des rôles institutionnalisés des familles communautaires total

5 la famille est trop isolée 
VALEURS FAMILIALES DE SUJETS FÉMININS NOVATEURS

TABLEAU 3

Les relations idéales parents-enfants

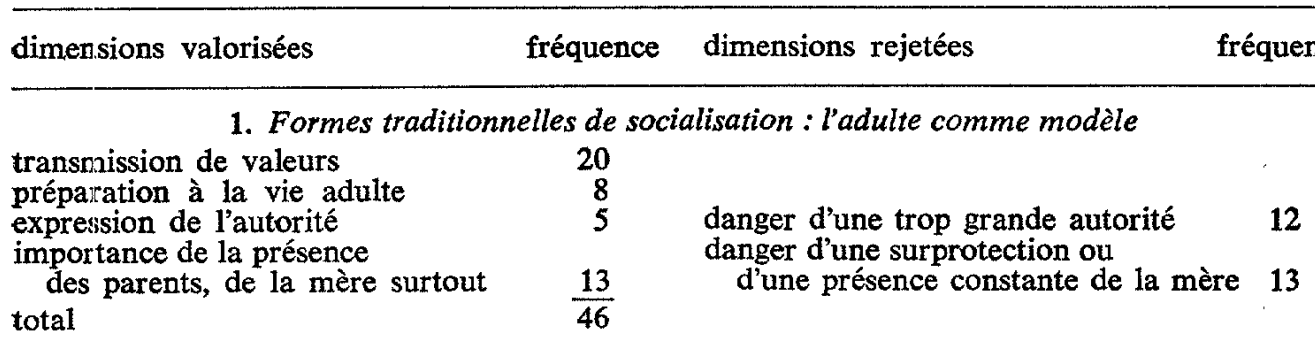

\section{Formes modernes : l'individualité comme priorité}

aspect: des tendances et

aptitudes individuelles

développement progressif

31

de l'autonomie

développement de la créativité

laisser:-faire

relations démocratiques

importance de l'information

partage des activités

disponibilité des parents

expression d'affection et

de sécurité émotionnelle

11

$$
14
$$

25

25

9 total

\section{$\frac{13}{135}$}

danger d'une trop grande liberté

laissée aux enfants

inégalité entre parents et enfants

séparation des activités

13

4

chaque génération développe

3. Possibilités nouvelles de développement

un nouveau modèle d'adulte

importance des différences

intergénérationnelles

authenticité des parents

échanges d'expériences

total

total positif

7
9
16
$\frac{12}{44}$
$\frac{225}{25}$

total négatif

$\overline{45}$

TABLEAU 4

Le mariage en tant qu'institution

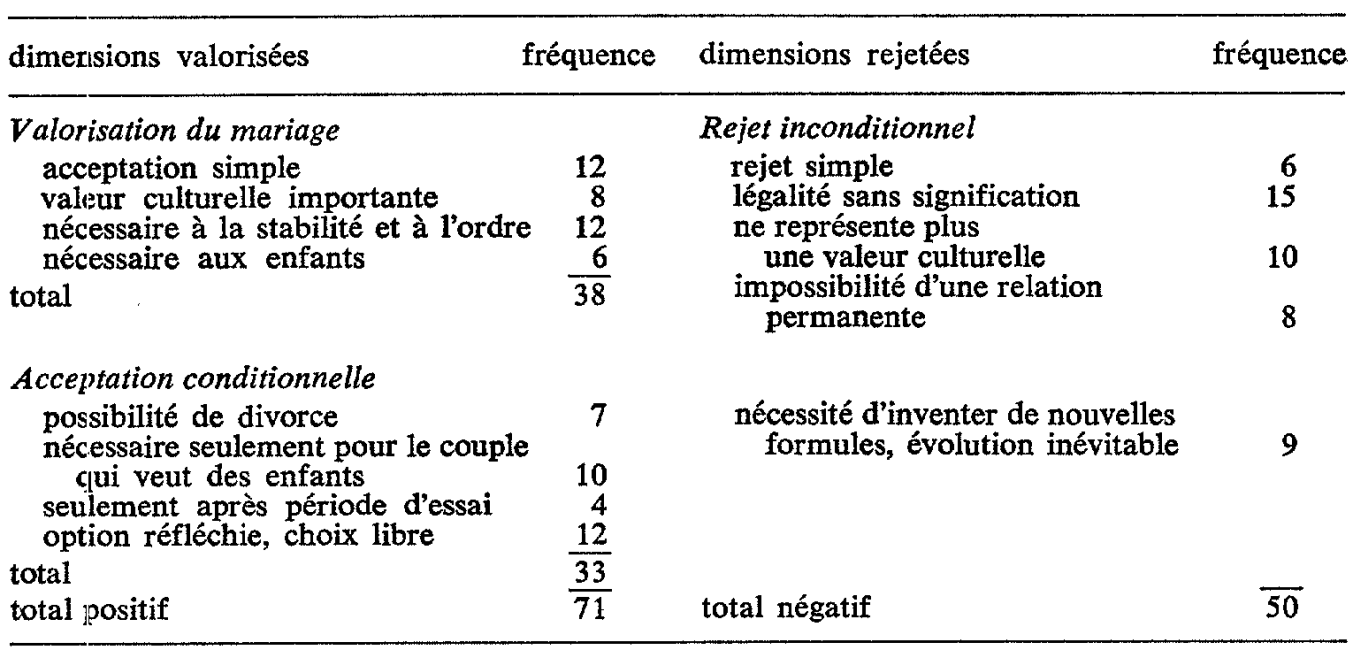


TABLEAU 5

Les relations idéales homme-femme

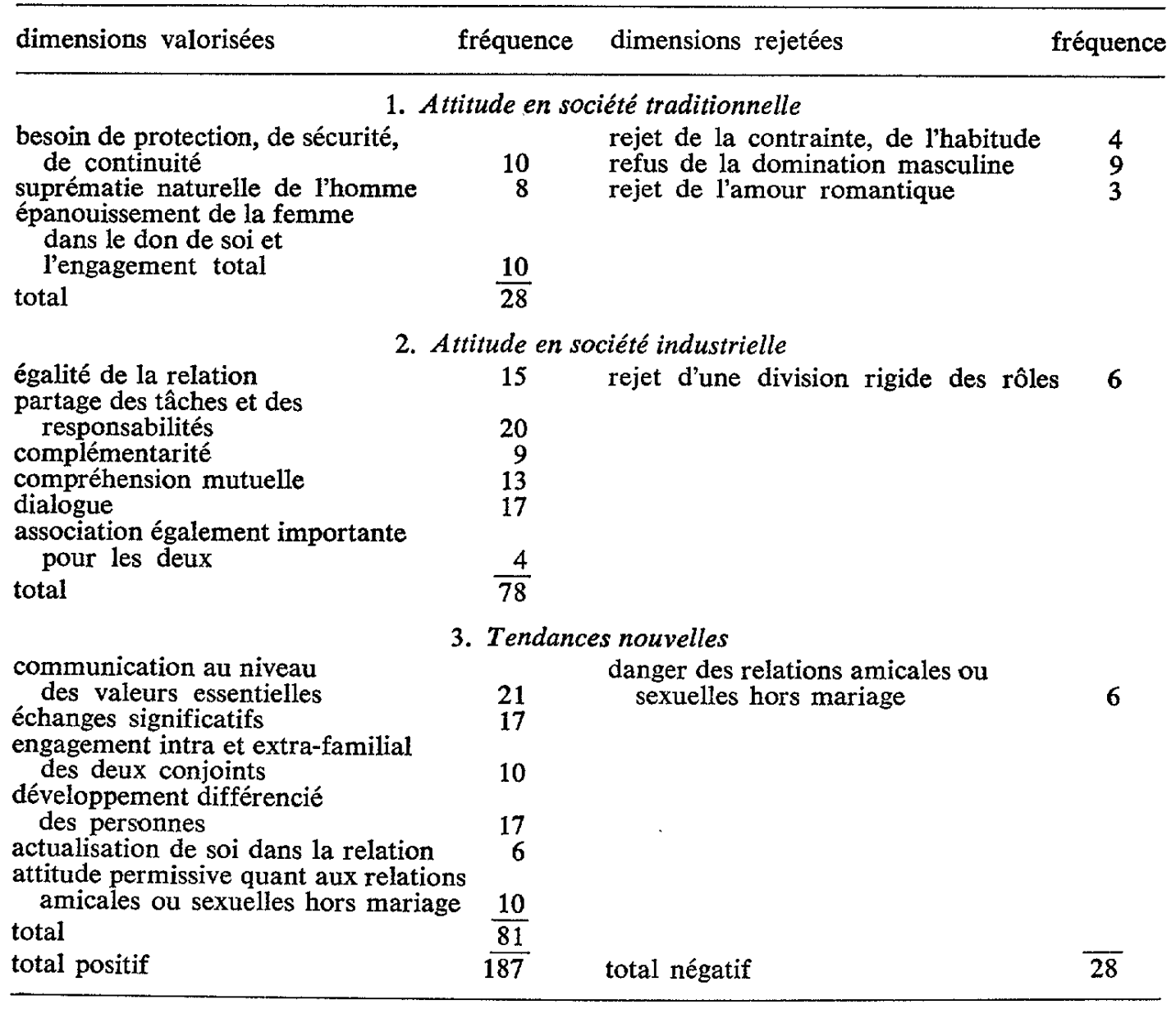

utilisant l'analyse contextuelle. Les fréquences qui apparaissent dans les quatre tableaux qui résument cette analyse se réfèrent donc à des mentions, non à des individus (voir tableaux 2, 3, 4 et 5). Nous appuyons sur le fait qu'il s'agit de réponses spontanées qui mettent en valeur ce que l'interviewée juge important, comme la transmission de certaines valeurs. Par contre, des aspects qu'on croit importants en sociologie de la famille, comme le système d'entraide ou la proximité écologique, n'ont pas été mentionnés une seule fois. Par voie de conséquence, les dimensions regroupées sous les trois points de référence ne constituent pas une description adéquate et complète de trois types de famille. Il s'agit carrément d'une exploration nécessaire à une recherche plus formelle.

Dans la description des résultats, nous avons procédé à un montage verbal des énoncés types. Certains entretiens étaient riches, d'autres bien pauvres. En fait, les réponses ont été plus souvent données en termes d'interrelation (questions 2 et 4 ) qu'en termes de contenu de l'institution (questions 1 et 3 ). Ce qui est important pour l'interprétation est la distribution des mentions. 


\section{LES RÉSULTATS}

\section{La famille comme institution (tableau 2)}

La famille, cellule de base de la société, est une définition classique et traditionnelle. La famille répond à « un besoin fondamental de la nature humaine [...] si l'on veut une nation équilibrée et travaillante, il faut développer les valeurs familiales ». «La vie familiale est source de joies profondes dans le présent et, au moment de la vieillesse, de satisfactions nettement supérieures aux réalisations professionnelles. \ La famille assure la continuité des générations dans la société et constitue « un cadre essentiel à l'épanouissement de l'enfant qu'on a créé ».

$\mathrm{Au}$ total, trente-huit mentions se réfèrent à ces dimensions traditionnelles de la famille-institution, contre vingt-neuf rejets qui explicitement mettent en doute l'importance ainsi accordée à la famille. Celle-ci est alors considérée comme inadaptée à la vie moderne, « elle est en train de mourir ». D'ailleurs, "d'autres influences, plus fortes que la famille, façonnent les enfants, leurs goûts, leurs aspirations». Et : "Pourquoi donc avoir des enfants dans cette société à l'envers? » On affirme alors le droit des couples de ne pas avoir d'enfants et d'utiliser leurs énergies à d'autres fins sociales ou personnelles.

Face au monde extérieur, urbain et industrialisé, impersonnel et compétitif, une femme sur cinq envisage la famille soit comme un refuge soit comme un lieu de ressourcement affectif. Cette perspective est éloquemment soulignée par une femme-médecin : "Les hommes sont pris dans la course... c'est le besoin de compétence. [...] C'est un monde dur, c'est un monde de technique, c'est un monde dépersonnalisant; où l'individu va-t-il trouver un havre où il peut être lui-même sans avoir peur de la concurrence, où il n'est pas pressé d'être différent de ce qu'il est, où est-ce sinon dans la famille ? »

L'image de la vie familiale n'est pas toujours perçue sous un angle aussi idyllique. Plusieurs soulignent la possibilité de conflit d'intérêts, surtout lorsque la fernme poursuit une carrière; aussi, la vie familiale doit-elle réaliser un équilibre entre les justes revendications de chacun de ses membres. Dans une même perspective, quelques femmes ont répondu à notre question en soulignant l'importance de planifier le nombre d'enfants. Mais en général, cette notion du foyer chaleureux où l'on se repose des luttes menées à l'extérieur n'est explicitement renié par aucune femme. Cette définition de la vie familiale est en quelque sorte négative, elle situe la famille par rapport à la vie trépidante de la société industrielle. Dans le même esprit, le loisir serait défini comme une détente qui permet de refaire ses forces pour mieux travailler après. Un dépassement de cette définition par la négative a été perçu par certaines femmes qui ont envisagé la vie familiale par le plaisir intrinsèque qu'elle procure. D'autres, enfin, rejettent l'idéologie qui valorise le rôle maternel aux dépens des autres rôles de la configuration féminine.

Des indicateurs de redéfinition de la vie familiale se retrouvent dans dix-huit mentions. L'essentiel de la vie familiale devient alors « l'invention d'un style de vie original 》 [...] « qui répond aux besoins d'expression des personnes 》 [...] « laissant la liberté à chacun ». La famille et les relations des membres de la famille sont envisagées comme des ressources qui favorisent l'actualisation de soi 
car « c'est l'individu qui est la cellule de base de la société et non la famille ». C'est ainsi que plusieurs rejettent, parfois violemment, l'institutionnalisation des rôles masculins et féminins et la division des tâches et de l'autorité qui en sont le corollaire ${ }^{16}$. Pour un dernier groupe, cette famille où l'on a cherché à se réfugier « se referme avec risque d'asphyxie ». "La famille doit éclater pour libérer l'individu. » On veut «vivre dans une communauté qui ne se réduit pas à une seule famille »; «créer des sous-groupes, des communautés où les gens parlent entre eux, créent une fraternité, où l'on peut s'aider, où on ne se sent pas perdu »; « où les enfants pourraient être élevés par plusieurs»; «où les rôles sont partagés. selon les aptitudes ».

On veut donc réduire la rigidité ou la formalisation dans les fonctions ou relations familiales pour favoriser un élément de proximité dans la relation dont le prototype est la relation amoureuse ${ }^{17}$.

On est donc bien loin de l'unanimité quand on demande à des femmes novatrices de donner leur conception de la famille. Trente-huit mentions se réfèrent à des éléments de la famille traditionnelle; quarante situent la vie familiale en regard de la vie urbaine dans une société industrielle; trente-deux rejettent carrément l'un ou l'autre de ces éléments de définition et quinze autres indiquent des tendances nouvelles de la vie familiale. On peut donc conclure que, sur cinq mentions, trois se situent dans les types connus, et deux rejettent ceux-ci ou se situent en deçà.

\section{La relation parents-enfants (tableau 3)}

Les sujets interrogés définissent le plus souvent le rôle des parents par leurs responsabilités vis-à-vis de l'enfant; le contenu de la relation peut donc le plus facilement être classifié selon les modes de socialisation qui sont envisagés. Nous avons distingué trois conceptions qui sont : traditionnelle, moderne ou postmoderne.

La première conception postule que l'adulte est un produit fini possédant une certaine fixité de croyance. L'enfant est donc « un adulte en préparation, un adulte provisoirement inachevé et incomplet $\gg$ et l'éducation consiste à transmettre d'une génération à l'autre les valeurs culturelles d'une civilisation dans une société et une classe donnée ${ }^{18}$. Quarante-neuf mentions relèvent de ce modèle qui implique à la fois la priorité de certaines valeurs et un mode de contrôle de l'adulte en herbe : «Il faut que les parents inculquent des principes à leurs enfants...»;

16. Pour Wilhelm Reich, la révolution russe n'a pas réussi à réaliser un socialisme humain parce qu'elle s'est attaquée seulement à l'hégémonie de classe en ignorant le fait que cette structure d'autorité se prolongeait dans les structures patriarcales de la famille traditionnelle qui lui servait de support (la Révolution sexuelle, Paris, \& Le monde en 10-18\%, 1970).

17. Robert Sévigny offre une typologie des interactions basée sur la distance psychologique entre l'individu et le groupe : a) secteur immédiat, impliquant les expériences des réseaux informels de communication, $v . g$. relation amoureuse ; $b$ ) secteur institutionnalisé où les relations sont définies par des codes sociaux, $v$. $g$. famille, religion, milieu de travail ; c) secteur des grands ensembles où les expériences sont directement reliées aux éléments de structures sociales, v. $g$. ville, classe, ethnie. La famille, dans ce contexte, ne peut être définie comme groupe primaire parce que très institutionnalisée; nous sommes totalement d'accord avec cette position (cf. Sociologie et Sociétés, vol. 1, $\mathrm{n}^{\circ} 2$, novembre 1969, p. 201).

18. Nous nous sommes inspirés, dans cette classification, de l'analyse de Lapassade qui lui-même utilise la critique que fait E. Pichon de Piaget (cf. l'Entrée dans la vie, Paris, Editions de Minuit, 1963, p. 198). 
«il $\mathrm{y}$ a en nous une certaine morale qu'il faut suivre». Les valeurs les plus souvent mentionnées dans ce contexte sont le sens des responsabilités, l'amour du travail, la discipline, la justice, la possibilité de se fixer des objectifs et de décider, le respect des autres, la tendresse, mais aussi le rejet de la priorité accordée au travail, de la concurrence et d'une définition matérialiste du succès. «Grandir, c'est se créer des outils, des outils pour vivre. » Pour atteindre cet objectif de socialisation, les parents doivent exercer une saine autorité et leur présence au foyer, celle de la mère surtout, est jugée primordiale. Par contre, dix-neuf mentions soulignent les dangers de la surprotection et des abus d'autorité.

Selon la conception traditionnelle de l'éducation, il existe une définition culturelle de l'adulte "normal » et la maturité consiste à réaliser cet idéal. Dans une société industrielle basée sur l'individualité et le succès, ce monolithisme traditionnel se brise et il existe différents modèles. Chaque enfant peut se développer selon ses propres ressources. Un nombre important de mentions (140) relèvent du principe d'individualité, chaque enfant devant se développer selon ses ressources propres sans référence à un modèle d'homme adulte. On peut dire qu'on prépare des individus qui devront acquérir leur statut dans une société compétitive basée sur l'achievement : «Si on s'habitue à voir un enfant, même tout petit, comme un individu autre que nous-mêmes, comme un futur adulte qui s'en va vers sa propre maturité à lui, qui a ses idées à lui, alors on acceptera qu'il ne soit pas la réplique de ses parents. \$ Pour certaines, «l'expérience est incommunicable » et «l'enfant apprendra en se faisant taper sur les doigts». Pour d'autres, le respect de la personnalité des enfants n'est pas synonyme de démission des parents; il faut au contraire « aider l'enfant à développer ce point de la création, de l'originalité et de la personnalité »; «il faut l'aider à devenir de plus en plus autonome »; 《il fauut donner à l'enfant les moyens d'exercer sa liberté ». À cette fin, l'importance de l'information éclairée et abondante est soulignée; cette information est essentielle à une relation démocratique, où les décisions sont prises en commun et les activités partagées. Pour se développer, l'enfant devra se sentir protégé et aimé. Devant cette faveur dominante accordée à l'individualité, certaines femmes mettent en garde contre une trop grande liberté accordée à l'enfant, ou encore soulignent que parents et enfants ne peuvent être considérés comme des égaux.

Les deux premiers modèles postulent que l'état d'adulte est la finalisation d'un processus. Le troisième modèle, tout en rejetant le concept d'adulte-étalon, va plus loin que la notion d'individualité propre à chacun, sans la renier toutefois. L'enfant annoncerait l'adulte à venir, non plus sur le plan individuel, mais dans l'histoire collective. C'est la notion de néoténie qui postule que les nouveaux courants intellectuels passent par les générations nouvelles; c'est le rôle des adultes de percevoir ces courants et d'aider à les actualiser. C'est une forme de socialisation à rebours, qui complète la première dans un système d'échanges intergénérationnels. Aucune des femmes novatrices que nous avons interrogées ne décrit les relations parents-enfants explicitement sous cette forme : toutefois plusieurs éléments de définition se retrouvent dans des expressions comme celles-ci : «... les enfants vivent dans un monde différent du nôtre et ne suivront pas les mêmes itinéraires »; "sur bien des plans les enfants nous dépassent». Devant cette différence intergénérationnelle, plusieurs affirment le besoin d'être d'abord \& authentiquement soi-même, sans cacher ce qu'on considère des défauts, des lacunes ». 
«C'est seulement en étant soi-même très vrai qu'on peut avoir une vraie communication avec les jeunes, » Une fois admise la différence essentielle entre générations, on peut alors parler d'échange d'expérience, tel que souligné par plusieurs : "éduquer mes enfants a voulu dire m'éduquer moi-même»; «j'ai appris à être femme au contact de mes filles »; « j'ai redécouvert la frâtcheur de la vie en jouant avec mon fils ». La relation parent-enfant peut donc être envisagée comme un processus d'échange dans une actualisation permanente de soi, tant des adultes que des enfants.

En résumé, la moitié des mentions favorisent l'individualité et le développement des ressources individuelles, un tiers craignent de laisser à l'enfant une trop grande liberté ou optent pour le modèle traditionnel de la transmission des valeurs sociales. Un sixième reconnaissent à la jeune génération des traits nouveaux que l'adulte peut capter et intégrer dans un dépassement des stades de développement déjà atteints chez la personne adulte.

\section{Le mariage comme institution (tableau 4)}

Est-ce que famille veut dire mariage ? Pas nécessairement, comme le dit une femme novatrice, "dans mon couple idéal, pas marié mais qui vit ensemble et qui a des enfants... ${ }^{19}{ }^{»}$.

Le mariage, institution qui formalise la conjugalité sur une base permanente, est accepté comme tel par relativement peu de femmes novatrices, soit au total par trente-huit. Pour celles-ci, le mariage est une institution valable, qui fait partie de notre culture, qui est nécessaire à une vie stable tant pour les adultes que pour les enfants.

L'acceptation de l'institution conjugale est conditionnelle pour un nombre équivalent de femmes, soit trente-trois : «Le mariage est nécessaire seulement si un couple veut avoir des enfants. $\gg$ On ne saurait non plus décréter que l'union sera permanente : le divorce doit être possible. Pour d'autres, la société ne saurait imposer le mariage à un couple : ce devrait être un acte librement consenti, un choix lucide, préférablement après essai.

Le refus du mariage-institution peut être brutal : « Je ne me marierais que pour avoir le statut de divorcée $\gg$ nous dit une jeune célibataire, mère d'un enfant qu'elle a voulu. Le mariage n'est qu'une formalité sans signification pour le couple. C'est une coutume en voie de disparition, qui donne une fausse sécurité, surtout à la femme : «Les gens s'installent dans le mariage...» «Ma conclusion, c'est que le mariage, comme institution, crée une situation psychologique qui fait qu'on est sûr d'un autre, alors on ne se remet pas en question. Je pense que dans l'amour, il y a ce choix de tous les matins qui est extrêmement important.»

Il se peut fort bien que le mariage-institution soit rejeté par une forte proportion des femmes novatrices à cause d'exigences plus grandes, d'un désir d'authenticité qui fera rechercher des formes nouvelles de relations.

19. Cinquante-deux pour cent des femmes interrogées connaissent présentement un mariage stable, sans échec antérieur; la presque totalité ont des enfants. Un autre quatorze pour cent a connu un échec conjugal qui s'est soldé par une séparation ou un divorce. Près d'un tiers n'ont jamais été mariées. 


\section{Relation idéale homme-femme (tableau 5)}

Nous n'avons pas justifié, au début de cet exposé, les raisons qui nous ont fait choisir d'étudier l'intervention sociale par des sujets féminins plutôt que masculins. Disons que dans une société caractérisée par les mutations culturelles, la redéfinition des rôles en termes d'universalité ou de spécificité masculine-féminine est primordiale. La femme cherche de nouvelles relations avec le monde : le jeu de sa propre liberté aura certainement une influence décisive sur le contenu culturel de nos sociétés avancées. L'étude des valeurs féminines dans l'action nous est donc apparue comme tout à fait primordiale dans une étude orientée vers l'avenir. Or, comme le souligne Memmi, la femme a été brimée, dans la loi et les mœurs, à partir de sa féminité même, telle que définie dans ses relations à l'homme et à l'enfant ${ }^{20}$. La présente analyse de la définition idéale des échanges et des besoins réciproques de l'homme et de la femme revêt donc une importance particulière.

À cause du nombre important de refus du mariage comme forme de conjugalité, la relation idéale entre homme et femme s'applique à tous les couples, mariés ou non. Il n'est pas facile d'établir les dominantes qui correspondent aux trois types de société; nous avons essayé d'être cohérents avec ce qui a été esquissé jusqu'ici. L'attitude traditionnelle, comme toujours, est plus facile à définir puisque le profil se détache sur un passé qui a été une réalité historique; cette attitude a défini la femme comme inférieure à l'homme et l'amour comme un don de l'être féminin à un homme naturellement actif et agressif.

La phase de la libération, qui correspond à l'ère industrielle, affirme par opposition l'individualité de la femme, ses droits, et l'aspect contractuel (bargaining) des accommodations. La troisième phase, celle de la liberté acquise, chercherait la signification de la relation dans la gratification, dans le contenu même de la communication qui est plus qu'un dialogue, et dans la relation comme telle qui est source d'actualisation de soi. Cette relation, très intense dans le présent, n'implique pas la permanence, sans toutefois la rejeter nécessairement.

Vingt-huit mentions seulement se réfèrent à une définition traditionnelle des rôles masculins et féminins : «Je considère que la femme a besoin de trouver la sécurité près de l'homme... »; «j'estime que c'est l'homme qui doit orienter la femme; quand j'aime un homme je veux que ça soit lui qui dirige »; «pour être heureuse dans le mariage, il faut que le mari soit supérieur »; «c'est en s'oubliant pour son mari et ses enfants que la femme va s'épanouir »; "quand une femme se marie elle doit tout abandonner en dehors de sa vie de femme, elle doit être amoureuse, elle doit être toute beauté, tout repos pour son mari ». Peu de femmes modernes feraient leurs ces affirmations bien que les femmes qui les ont prononcées n'en aient pas moins fait leur marque dans des secteurs précis d'activité. Seize femmes, par contre, rejettent explicitement ces mêmes attitudes.

20. A. Memmi, * Plaidoyer d'un tyran ", dans l'Homme dominé, Paris, Gallimard, 1968 , p. 155-171. Nous citons (p. 167) : or, la féminité est le lieu d'oppression de la femme; comme la négritude est le lieu d'oppression du Noir, et la judéité celui du Juif. Or, l'originalité de l'oppression de la femme est dans son rapport à l'homme et à l'enfant. Ai-je besoin de le répéter, la femme est victime de la société globale, certes, et dans toute sa conduite. Mais tout opprimé est atteint spécifiquement, d'une manière qui entraîne toutes les autres. Et pour agir au mieux sur une oppression particulière il faut d'abord mettre en lumière ses particularités. Pour être libérée efficacement, la femme doit être considérée comme femme : c'est-à-dire comme amante et mère. Pour libérer la femme, il faudra instaurer des rapports nouveaux dans les relations amoureuses et dans la maternité. » 
Nous avons dit que l'individualisme est une valeur de base en société industrielle; la relation entre homme et femme s'établit donc entre un homme libre et une femme libérée des servitudes traditionnelles. Cette égalité est la base de la relation pour quinze interviewées : «Je pose que l'homme et la femme sont des individus égaux et je pose aussi que vivre c'est se développer, c'est produire et créer. » Pour quelques-unes, cette égalité s'harmonise dans la complémentarité et est confirmée dans le partage des tâches et des responsabilités. Il peut s'agir d'un échange fonctionnel, tel que décrit par Parsons : rôle instrumental de l'homme, extérieur à la famille, rôle affectif de la femme, intérieur à la famille. Le plus souvent, il s'agit de collaboration à l'intérieur même du foyer afin que «les deux aient des chances égales », «qu'il n'y ait pas un conjoint qui soit plus surchargé que l'autre ». "Il s'agit d'un échange entre partenaires. » Et tout naturellement, on est amené à parler de dialogue et de compréhension, car \& un dialogue peut s'établir entre égaux». «Il ne faut pas se décourager s'il y a des problèmes, il y en aura toujours; la meilleure façon de s'entendre, c'est d'en discuter. » Partenaires égaux, dialogue et compréhension sont donc les mots clefs dans soixante-quatorze mentions.

Un troisième groupe de réponses se situe, nous semble-t-il, à un niveau d'intensité plus élevé. Non seulement on parle de dialogue mais aussi d'échanges dont le contenu est important et significatif pour les deux conjoints. II faut rappeler que les femmes novatrices que nous avons interrogées peuvent contribuer positivement à ces échanges à cause de l'intérêt même de leur activité. Comme le décrit une jeune célibataire «nous avons bâti un nouveau monde qui est une confrontation constante de ce que l'on vit $»$; «j'ai l'impression qu'un couple peut évoluer dans la mesure où l'un et l'autre se rattachent à des schèmes bien définis, à des valeurs partagées et aussi à des expériences vécues séparément et mises en commun ensuite ». Pour quelques-unes, il serait inconcevable de se lier à un homme qui n'est pas engagé lui-même dans une action qu'elles estiment importante. Non seulement on parle de compréhension, mais de communication \& au niveau des valeurs essentielles 》; "avoir les mêmes intérêts, les mêmes inspirations c'est ce qu'il y a de plus important ». "En fait, la richesse de la communication dépend du développement continu de la personnalité.» «Il faut que chacun s'engage avec la conviction que l'autre doit trouver vraiment le meilleur de lui-même; il faut donc qu'on soit prêt à découvrir des visages imprévisibles dans l'évolution de la personnalité de l'autre. »

Pour un dernier groupe, la relation amoureuse ne veut pas dire don de soi mais bien découverte de soi : «La relation du couple c'est passionnant, on vit toujours quelque chose de neuf. Si on vit seul, probablement qu'il y a une évolution aussi, mais c'est moins complexe. Il n'est pas question de se fondre à l'autre, il s'agit d'avoir un répondant valable à ses émotions, à ses idées, à son action. 》

Dans l'ensemble, donc, peu de femmes innovatrices acceptent l'attitude de soumission dans leur définition de la relation homme-femme. Plus d'un tiers affirme l'égalité des droits; près d'un tiers dépasse cette notion et souligne l'importance du contenu gratifiant de cette relation. 


\section{CONCLUSION}

$\mathrm{Au}$ total, 840 mentions se rapportant à la vie familiale et à la conjugalité ont été catégorisées selon les sociétés dont elles sont typiques : 535 mentions définissent une vie familiale qui relève de la société traditionnelle ou industrielle. Par contre, 305 mentions, ou bien rejettent ces mêmes définitions ou bien se situent en deçà, indiquant des dominantes de la vie familiale et conjugale en devenir. Du point de vue méthodologique, un système d'analyse qui laisse en plan quarante pour cent des données doit être repensé. Nous avons tenté, dans cet exposé, d'esquisser les grandes lignes d'un modèle plus complexe qui permettrait de confronter la dynamique familiale à la dynamique sociale. Un effort systématique pour: articuler la relation famille/société nouvelle mérite d'être tenté. Nous espérons le faire dans une recherche ultérieure explicitement dirigée dans ce sens.

La base traditionnelle de la vie familiale est facile à définir, mais dans l'ensemble, seulement une mention sur six s'y rattache. Selon cette conception, la famille a, comme première fonction, la transmission des valeurs qui feront de l'enfant un adulte dont les parents sont les modèles. La famille offre protection et sécurité. La femme trouve son épanouissement auprès de ses enfants et dans l'amour de son mari. L'autorité est ferme et hiérarchisée.

Près de la moitié des mentions se rattachent à un modèle qu'on peut dire moderne, axé essentiellement sur le développement individuel, sur des relations démocratiques, sur un partage des responsabilités qui ne pénalise aucun des membres du groupe familial ou conjugal. La famille est le lieu de repos face au monde du travail; les relations entre individus sont empreintes d'une affectivité qui est la contrepartie de la compétition en société industrielle.

La somme des mentions négatives qui rejettent ces formes institutionnalisées est relativement importante, soit une mention sur cinq. C'est le refus de la contrainte, de tout ce qui est figé dans les normes, dans la permanence, dans la définition rigide des rôles appris. La contrepartie se retrouve dans le désir d'inventer un style de vie, d'établir des relations basées sur l'échange d'expériences, sur la communication des valeurs, sur l'authenticité des personnes. Ce réseau de communications intenses doit préférablement s'étendre hors des cadres de la famille nucléaire. Parents et enfants, hommes et femmes, font face à un besoin de développement constant. Ces traits, nous semble-t-il, définissent une société où l'essentiel relève de mutations culturelles extrêmement rapides et souvent abruptes. C'est l'hypothèse émise au début de cet exposé, soit que l'ampleur des changements technologiques dans les sociétés développées a des répercussions qualitatives sur le style de vie, sur les normes et les valeurs qui sous-tendent la vie privée des personnes, la vie quotidienne des familles et des couples. Il nous semble donc que, dans des recherches futures, nous aurions profit à distinguer ce qui appartient à une société industrielle, technologiquement avancée, des éléments de redéfinition d'une famille en face d'une société nouvelle dont elle est partie intégrante. I devient alors impossible de parler en termes moralisateurs d'une désintégration regrettable de la famille.

En corollaire, on peut se demander jusqu'à quel point les dimensions révélées et classifiées par nous comme postindustrielles sont en fait annonciatrices 
d'une vie familiale de l'avenir. Celui qui innove crée des modèles culturels nouveaux; quelle est la relation entre ces modèles et des changements probables ou possibles dans la vie familiale considérée comme un secteur de vie ? Peut-être serait-il préférable d'affirmer que ces individus sont des déviants dans un système d'ordre. Ces femmes sont-elles des éléments pervertissants qui minent les valeurs familiales et poussent la famille et le mariage à leur désintégration ? La façon dont nous avons formulé la recherche ne permet de répondre ni à l'une ni à l'autre de ces questions qui expriment, par leur formulation même, des prises de position idéologiques. Par contre, les femmes interrogées dans cette recherche sont bien éduquées, localisées près des sources d'information, elles ont en moyenne trois enfants et participent à des activités professionnelles ou sociales. Elles sont donc à l'avant-garde en ce sens qu'elles représentent des tendances observables dans notre société. De plus, en tant qu'innovatrices, nous avons présumé que leur niveau de conscience sociale est nettement plus élevé que celui de l'ensemble de la population. Leur définition d'une situation doit donc être prise au sérieux, voire même considérée avec un préjugé favorable. Ce qu'elles disent ou réalisent constitue en fait une intervention dans la réalité sociale. Cette intervention appellera une réaction qui pourra être renforcement, opposition ou réinterprétation. Un changement peut être le résultat de ce processus qui n'est pas linéaire mais circulaire, impliquant une redéfinition constante de la situation. L'importance $d u$ changement dépendra de l'ouverture au changement de l'ensemble de la population et des sous-groupes. Une expérience récente nous a par ailleurs convaincus que l'attente de changement dans la vie familiale est très élevée et que ce changement est fortement valorisé dans les groupes d'âge en bas de quarante ans ${ }^{21}$. La direction de ce changement dépend largement de la diffusion accordée aux valeurs des novateurs. Or, une récente analyse de 3500 événements rapportés dans la presse écrite et dont l'acteur est féminin montre clairement que les activités novatrices sont plus «visibles» en 1967 qu'en 1954. De plus, les modèles culturels nouveaux des novatrices sont diffusés avec une intensité qui augmente avec le niveau culturel du public à qui l'on s'adresse ${ }^{22}$. En dernier lieu, il est important de souligner qu'une politique familiale peut favoriser, modifier ou freiner ce mouvement. Une politique réaliste (qu'on oppose à une position moralisatrice) doit tenir compte de cette interaction entre les éléments de la réalité sociale.

RÉSUMÉ

Le présent article essaie d'esquisser les tendances du changement familial dans une société technologiquement avancée. Les données ont été recueillies auprès de cent cinquante femmes jugées novatrices dans divers secteurs d'activité. Nous leur avons demandé : 1) Que pensezvous de la famille comme institution? 2) Quelle serait la relation idéale parents-enfants? 3) Que pensez-vous du mariage ? 4) Quelle serait la relation idéale entre un homme et une femme? Les résultats ont été regroupés à partir de trois points de référence qui correspondent aux trois types de société : traditionnelle, industrielle et postindustrielle. Près des deux tiers des mentions définissent une vie familiale qui relève de la société traditionnelle ou industrielle.

21. Impression que nous a laissée la lecture des lettres (2000) répondant à la question suivante : "En quoi la famille de vos enfants sera-t-elle différente de la vôtre ou de celle de vos parents? (Femmes d'aujourd'hui, magazine féminin d'une heure présenté à la télévision d'Etat chaque jour de la semaine, saison 1970).

22. C. Carisse, «Image de l'action féminine », texte présenté au deuxième congrès de 1'I. C. O. F. A., Rennes, avril 1969, et publié en traduction anglaise dans les Actes du congrès. 
Par contre, les autres ou bien rejettent ces définitions ou bien se situent en deçà indiquant des dominantes de la vie familiale ou conjugale en devenir.

\section{ABSTRACT}

[The Family Values of Innovating Feminine Subjects : Perspectives of the Future] This is an atternpt to sketch trends in family change in a technologically advanced society. The data were collected from one hundred and fifty women judged to be innovators in different sectors of activity. We asked them : 1) What do you think of the family as an institution ? 2) Which would be the ideal relationship between parents and children? 3) What do you think of marriage ? 4) Which would be the ideal relationship between a man and a woman ? The results were grouped according to three reference marks corresponding to three types of societies : traditional, industrial and postindustrial. Nearly two thirds of the replies define family life typical of traditional or industrial society; the rest either reject these definitions or leave them aside indicating the main principles of family or conjugal life to come.

\section{RESUMEN}

[Valores familiares de sujetos femeninos innovadores : perspectivas de futuro] El presente artículo trata de bosquejar las tendencias del cambio familiar en una sociedad technológicamente avarizada. Los datos fueron recogidos por medio de entrevistas a ciento cincuenta mujeres consideradas como innovadoras en diversos sectores de actividad. Se les ha preguntado : 1) ¿Qué piensa Usted de la familia como institución? 2) ¿Cuál sería la relación ideal entre padres e hijos? 3) ¿Qué piensa Usted del matrimonio? 4) ¿Cuál sería la relación ideal entre un hombre y una mujer ? Los resultados fueron regrupados a partir de tres puntos de referencia que corresponden a los tres tipos de sociedad : tradicional, industrial y postindustrial. Cerca de dos tercios de las menciones definen una vida familiar típica de la sociedad tradicional o industrial. Por el contrario, las otras, o bien rechazan esas definiciones, - bien se situan más acá de ellas, indicando ciertas líneas dominantes de la vida familiar o conyugal en devenir. 